\title{
CONSTRUCTIONS FOR ARC-TRANSITIVE DIGRAPHS
}

\author{
MARSTON CONDER, PETER LORIMER and CHERYL PRAEGER
}

(Received 4 April 1991; revised 1 October 1992)

Communicated by Louis Caccetta

\begin{abstract}
A number of constructions are given for arc-transitive digraphs, based on modifications of permutation representations of finite groups. In particular, it is shown that for every positive integer $s$ and for any transitive permutation group $P$ of degree $k$, there are infinitely many examples of a finite $k$-regular digraph with a group of automorphisms acting transitively on $s$-arcs (but not on $(s+1)$-arcs), such that the stabilizer of a vertex induces the action of $P$ on the out-neighbour set.
\end{abstract}

1991 Mathematics subject classification (Amer. Math. Soc.): 05C25, 20 B25.

\section{Introduction}

In this paper we describe a number of constructions for finite arc-transitive digraphs, including those which are $s$-arc-transitive for large values of $s$. In particular, we show how permutation representations of finite groups can be modified in various ways so as to produce infinitely many $s$-arc-transitive $k$-regular digraphs for every positive integer $s$ and for every integer $k \geq 2$.

This situation is quite different from that of finite undirected regular graphs of degree greater than 2, which by a theorem of Richard Weiss [7] can be at most 7-arc-transitive.

A digraph (short for directed graph) $\Delta$ may be regarded as a pair $(V, E)$ consisting of a set $V$ and a subset $E$ of the Cartesian product $V \times V$ : the members of $\mathrm{V}$ are the vertices of $\Delta$ and any member $(v, w)$ of $E$ is an edge from the vertex $v$ to the vertex $w$ in $\Delta$. Often the definition of a directed graph allows the possibility of multiple edges between a pair of vertices, but ours will not. The out-degree (respectively indegree) of a vertex $v$ is the number of vertices $w$ for which $(v, w) \in E$ (respectively $(w, v) \in E)$. An automorphism of a digraph $\Delta=(V, E)$ is defined to be any

(C) 1995 Australian Mathematical Society 0263-6115/95 \$A2.00+0.00 
permutation $\pi$ of the vertices which induces a permutation of the edges, that is, such that $\left(v^{\pi}, w^{\pi}\right) \in E$ whenever $(v, w) \in E$. Under composition of permutations, the set of all automorphisms of $\Delta$ forms a group, called the automorphism group of $\Delta$, and denoted by Aut $\Delta$. The edges of $\Delta$ may also be called arcs, and $\Delta$ is said to be arc-transitive if its automorphism group acts transitively on $E$. More generally, an $s$-arc in $\Delta$ is any sequence $\left(v_{0}, v_{1}, v_{2}, \ldots, v_{s}\right)$ of vertices of $\Delta$ such that $\left(v_{j-1}, v_{j}\right)$ is an edge of $\Delta$ for $1 \leq j \leq s$, and $\Delta$ is said to be $s$-arc-transitive if its automorphism group acts transitively on the set of all $s$-arcs in $\Delta$. It is clear that $s$-arc-transitivity always implies $(s-1)$-arc-transitivity, 1-arc-transitivity is the same as arc-transitivity, and, provided every vertex is incident to at least one edge, arc-transitivity implies vertextransitivity: the automorphism group of $\Delta=(V, E)$ is transitive on $V$. In particular, a vertex-transitive digraph is regular : every vertex has the same in-degree and the same out-degree; and if these have the common value $k$ (which happens for instance when the digraph is finite), the digraph is regular of degree $k$, or simply $k$-regular.

For example, a simple circuit digraph made up of vertices $1,2,3, \ldots, n$ and edges $(1,2),(2,3), \ldots(n-1, n),(n, 1)$, with cyclic automorphism group of order $n$, is a 1-regular digraph which is $s$-arc-transitive for every positive integer $s$. Insertion of new edges $(1,3),(2,4), \ldots(n-2, n),(n-1,1),(n, 2)$, however, produces a 2-regular digraph which is vertex-transitive but no longer arc-transitive (for $n \geq 4$ ).

Fundamental to every construction we shall give in this paper is the relationship between arc-transitivity and properties of double cosets of a vertex-stabilizer in the automorphism group. Although this relationship is part of the folk-lore of algebraic graph theory, we describe it here for the sake of completeness.

THEOREM. Suppose $\Delta=(V, E)$ is a finite digraph with arc-transitive automorphism group $G$. For any particular vertex $u \in V$, let $H=\left\{h \in G \mid u^{h}=u\right\}$, the stabilizer of $u$ in $G$, and define $D=\left\{g \in G \mid\left(u, u^{g}\right) \in E\right\}$. Then

(1) $D=H a H$ for some $a \in G$, and

(2) there is a one-to-one correspondence between vertices of $\Delta$ and right cosets of $H$ in $G$ given by $u^{g} \longleftrightarrow H g$ for all $g \in G$, such that

(3) the ordered pair $(H x, H y)$ corresponds to an edge of $\Delta$ if and only if $y x^{-1} \in D$, and

(4) the degree of $\Delta$ is equal to $\left|H: H \cap a^{-1} H a\right|$, the number of right cosets of $H$ in $D$, and

(5) $\Delta$ is connected if and only if $D$ generates $G$.

PROOF. First, if $a \in D$ then $H a H \subseteq D$ since $\left(u, u^{f a h}\right)=\left(u, u^{a h}\right)=\left(u^{h}, u^{a h}\right)=$ $\left(u, u^{a}\right)^{h} \in E$ for all $f, h \in H$. Also by arc-transitivity, whenever $\left(u, u^{g}\right) \in E$ there is some $h \in G$ such that $\left(u, u^{g}\right)=\left(u, u^{a}\right)^{h}=\left(u^{h}, u^{a h}\right)$, in which case $h \in H$ and $g(a h)^{-1} \in H$ (since $u=u^{h}$ and $u^{g}=u^{a h}$ ), so that $g \in H a h \subseteq H a H$. Thus (1) 
holds. Assertion (2) is a standard consequence of the transitive action of a group on a set : $u^{f}=u^{g}$ if and only if $f g^{-1} \in H$, that is, if and only if $H f=H g$. Next $\left(u^{x}, u^{y}\right) \in E$ if and only if $\left(u, u^{y x^{-1}}\right)=\left(u^{x}, u^{y}\right)^{x^{-1}} \in E$, that is, if and only if $y x^{-1} \in D$, giving (3). Also, the out-degree of the vertex $u$ is equal to the number of cosets of the form $H y$ with $y \in D$, which is simply the number of right cosets of $H$ in $D(=H a H)$. Cosets $H a f$ and $H a h$ are equal if and only if $a f h^{-1} a^{-1} \in H$, in which case $f h^{-1} \in H \cap a^{-1} H a$, and so this number is equal to the index of $H \cap a^{-1} H a$ in $H$. Again by arc-transitivity, every vertex has this degree, proving (4).

Finally, suppose the vertices corresponding to cosets $H x$ and $H y$ in $\Delta$ are joined by a path of the form $\left(H x=H x_{0}, H x_{1}, H x_{2}, \ldots, H x_{t-1}, H x_{t}=H y\right)$ with either $\left(H x_{j-1}, H x_{j}\right) \in E$ or $\left(H x_{j}, H x_{j-1}\right) \in E$ for $1 \leq j \leq t$. Then for all such $j$ either $x_{j} x_{j-1}^{-1} \in D$ or $x_{j} x_{j-1}^{-1}=\left(x_{j-1} x_{j}^{-1}\right)^{-1} \in D^{-1}$, so that $y x^{-1}=\left(x_{t} x_{t-1}^{-1}\right)\left(x_{t-1} x_{t-2}^{-1}\right) \cdots$ $\left(x_{2} x_{1-1}\right)\left(x_{1} x_{0}^{-1}\right)$ is a product of elements of $D \cup D^{-1}$. It follows that if $\Delta$ is connected, then $D$ generates $G$. The converse is also true, for if any element $g$ of $G$ is expressible as a product of elements of $D$ or their inverses, then the vertices corresponding to $H$ and $\mathrm{Hg}$ in $\Delta$ are joined by a path of the above form. Thus we have (5), and hence the theorem.

It is perhaps worth noting here that properties (2), (3) and (5) are held also by digraphs which are vertex-transitive but not necessarily arc-transitive, and in each case $D$ is a union of double cosets of $H$ and the degree remains equal to the number of right cosets of $H$ in $D$.

On the other hand, for undirected arc-transitive graphs an additional property is satisfied, namely $a^{2} \in H$ (as the element a may be chosen so that $(u, v)^{a}=(v, u)$ for some vertex $v$ adjacent to $u$ ).

More importantly, the theorem gives rise to the following.

General construction. For any finite group $G$ containing a subgroup $H$ and an element $a$ of $G$ such that $a^{-1} \notin H a H$, let $V$ be the set of all right cosets of $H$ in $G$, and define $E=\left\{(H x, H y) \mid y x^{-1} \in H a H\right\}$. Then $\Delta=(V, E)$ is a digraph, on which $G$ acts as a group of automorphisms via the rule $g: H x \mapsto H x g$ for all $x \in G$ and all $g \in G$. In particular, the subgroup $H$ is the stabilizer in $G$ of the vertex $H$ (itself), and the action of $G$ is transitive on the arcs of $\Delta$. The degree of $\Delta$ is equal to $\left|H: H \cap a^{-1} H a\right|$, and $\Delta$ is connected if and only if $\langle H, a\rangle=G$.

We will denote this digraph by $\Delta(G, H, a)$. The proof that the construction works is straightforward and left to the reader. Note that the subgroup $\mathrm{H}$ acts transitively on the vertices of the form $H a h$ (with $h \in H$ ), and also that $\langle H, a\rangle=\langle H a H\rangle$ since $h=a^{-1}(a h)$ and clearly $a \in H a H$. The kernel of the action of $G$ on $\Delta$ is the intersection of all conjugates $x^{-1} H x$ of $H$ in $G$. In particular, the action of $G$ is faithful if and only if this intersection is trivial. The condition that $a^{-1} \notin \mathrm{HaH}$ (which may be restated either as $H a^{-1} H \neq H a H$ or as $H \cap a H a=\emptyset$ ) ensures that 
$\Delta$ cannot be viewed as an undirected graph, but of course if this is relaxed (to simply $a \notin H$ ) then the construction can be used for undirected graphs as well.

One motivation for the research we now report on was the following question, considered also by Peter Cameron in his D. Phil. thesis [1].

Does there exist a small (finite) 6-regular digraph $\Delta$ with a 2-arc-transitive group of automorphisms, such that the stabilizer of any particular vertex $v$ is isomorphic to the alternating group $A_{6}$, and the stabilizer of any $\operatorname{arc}(v, w)$ is isomorphic to the alternating group $A_{5}$, inducing the natural action of $A_{5}$ on the other five outneighbours of $v$, while at the same time acting transitively (as the group $P S L_{2}(5)$ ) on the six in-neighbours of $v$ ?

We shall construct a number of such digraphs in this paper, the smallest of which has 4096 vertices. The various methods of construction are dealt with separately in the following sections.

\section{2. 'Extra point' constructions}

One of the easiest ways to obtain an arc-transitive digraph is described in the example below.

Example 1. For any integer $k \geq 2$, let $H$ be the subgroup of the symmetric group $S_{2 k}$ of degree $2 k$ generated by the permutations $x=(1,2,3, \ldots, k)$ and $y=(k+1, k+$ $2, k+3, \ldots, 2 k$ ). Clearly $x$ and $y$ commute, so that $H$ is the direct product of two cyclic groups of order $k$, having two distinct orbits each of size $k$. Next add an extra point, say $2 k+1$, and define the permutation $a=(1, k+1,2 k+1)(2, k+2)(3, k+3) \ldots(k, 2 k)$. The three permutations $x, y$ and $a$ together generate a transitive subgroup $G$ of the symmetric group $S_{2 k+1}$, indeed of the alternating group $A_{2 k+1}$ whenever $k$ is odd. But further, as $x, y$ and $a^{3}$ all fix the point $2 k+1$, it is easy to see that $G$ is 2-transitive and therefore primitive on $\{1,2,3, \ldots, 2 k, 2 k+1\}$. Then since $a^{2}$ is a single 3-cycle (fixing the other $2 k-2$ points), the group $G$ is $S_{2 k+1}$ when $k$ is even, and $A_{2 k+1}$ when $k$ is odd, by [8; Theorem 13.3].

Note that $a^{-1} \notin H a H$, because $a^{-1}$ takes the point $2 k+1$ to the point $k+1$ while every element of the double coset $\mathrm{HaH}$ takes $2 k+1$ to one of the points of the set $\{1,2,3, \ldots, k\}$. In fact this particular choice for the element $a$ was made so that $a^{-1} x a=y$, while on the other hand $a^{-1} x^{i} y^{j} a \notin H$ whenever $y^{j}$ is non-trivial (as in that case $a^{-1} x^{i} y^{j} a$ moves the point $2 k+1$ ). For these reasons $H \cap a^{-1} H a=\langle y\rangle$, of index $k$ in $H$, and it follows that the intersection of all the conjugates of $H$ in $G$ is trivial.

By the general construction given earlier, $\Delta(G, H, a)$ is a connected $k$-regular digraph on $(2 k+1) ! / k^{2}$ or $(2 k+1) ! / 2 k^{2}$ vertices, depending on whether $k$ is even or odd, with $G$ as an arc-transitive group of automorphisms. (In fact the stabilizer in $G$ 
of a 2-arc of $\Delta(G, H, a)$ is isomorphic to $H \cap a^{-1} H a \cap a^{-2} H a^{2}$, which is trivial, so the action of $G$ is 2-arc-regular.)

This technique of adding an extra point may be taken further: if $n$ is any odd positive integer, and $a$ is taken as the permutation $(1, k+1,2 k+1,2 k+2, \ldots, 2 k+n)(2, k+$ $2)(3, k+3) \cdots(k, 2 k)$, then the group $G$ generated by $x, y$ and $a$ is either $S_{2 k+n}$ or $A_{2 k+n}$ (depending on whether $k$ is even or odd). Here in each case the stabilizer of the point $2 k+n$ contains $x, y$ and $a^{n+2}$ along with $a^{-j} x a^{j}$ for $1 \leq j \leq n$, and $x^{-1} a^{-2} x a^{2}$ is a 3-cycle (fixing the other $2 k+n-3$ points). Again by considering what happens to the point $2 k+1$, it is easy to verify that $a^{-1} \notin H a H$ and that $H \cap a^{-1} H a=\langle y\rangle$, and so on. Hence there are infinitely many 2 -arc-transitive $k$-regular digraphs of this type for every integer $k \geq 2$.

Adding an even number of extra points works just as well, except that for each even $n$ the group $G$ is an imprimitive subgroup of $S_{2 k+n}$, with $\{1,2, \ldots, k, 2 k+1,2 k+$ $3, \ldots, 2 k+n-1\}$ and $\{k+1, k+2, \ldots, 2 k, 2 k+2,2 k+4, \ldots, 2 k+n\}$ as blocks of imprimitivity. If $k$ is odd then $G$ is an extension of the direct product of two copies of the alternating group $A_{k+n / 2}$ (with orbits the blocks of imprimitivity for $G$ ) by an involutory automorphism which swaps the two factors. On the other hand, if $k$ is even then $G$ is much the same as in the odd case except that it contains also all the elements of the direct product of two copies of the symmetric group $S_{k+n / 2}$ which have even parity overall. Verification of these claims is left to the reader.

Incidentally (although this has very little to do with graph theory), the above example gives rise to a negative answer to the question of whether a common transversal can always be found for double cosets $K g L(g \in G)$ and $L g K(g \in G)$ of subgroups $K$ and $L$ in a group $G$. The first author is grateful to Stephen Glasby for bringing this question to his attention.

Let $x, y, H, a$ and $G$ be as before, and take $K=\langle x\rangle$ and $L=\langle y\rangle$, commuting subgroups of order $k$. Then $a^{-1} x a=y$, so that $x a=a y$, but on the other hand $a^{-1} y^{j} a \notin H$ unless $y^{j}$ is trivial, and from this it follows that the double coset $H a H$ contains exactly $k^{3}$ elements, each uniquely expressible in the form $y^{p} a x^{q} y^{r}$ with $p, q, r \in\{0,1, \ldots, k-1\}$. (Note: if $y^{p} a x^{q} y^{r}=y^{s} a x^{t} y^{u}$ with $p \neq s$ then $a^{-1} y^{p-s} a=x^{t-q} y^{u-r} \in H$, which is impossible.)

Now there are $k^{2}$ double cosets of the form $K y^{p} a x^{q} y^{r} L$, each containing $k$ elements (of the form $y^{p} a x^{q} y^{m}$ where $m$ is variable, as $x^{i}\left(y^{p} a x^{q} y^{r}\right) y^{j}=y^{p} x^{i} a x^{q} y^{r+j}=$ $y^{p} a y^{i} x^{q} y^{r+j}=y^{p} a x^{q} y^{i+r+j}$ ), while on the other hand there are $k$ double cosets of the form $L y^{p} a x^{q} y^{r} K$, each of size $k^{2}$ (with all elements of the form $y^{m} a x^{n} y^{r}$ where $m$ and $n$ are variable, as $\left.y^{j}\left(y^{p} a x^{q} y^{r}\right) x^{i}=y^{j+p} a x^{q+i} y^{r}\right)$. In particular, any common transversal for the double cosets $K g L(g \in G)$ and $L g K(g \in G)$ would need to contain $k^{2}$ representatives from $\mathrm{HaH}$ for those of the first sort, yet only $k$ of these could be representatives for those of the second sort! As an illustration: in the case $k=2$ the double cosets of the form $K g L$ include $\{a, a y\},\{a x, a x y\},\{y a, y a y\}$ and 
\{yax, yaxy\}, but the eight elements in the union of these fall into just two double cosets of the form $\operatorname{Lg} K$, namely $\{a, a x, a, y a x\}$ and $\{a y, a x y, y a y, y a x y\}$.

Hence no common transversal exists (for any value of $k \geq 2$ ).

Returning now to the subject of this paper, we generalize the situation described in Example 1 by taking a direct product of a larger number of cyclic groups, to obtain the following result.

THEOREM 1. For every integer $k \geq 2$ and every positive integer $s$, there are infinitely many finite $k$-regular digraphs that have an $s$-arc-regular group of automorphisms.

A similar theorem was proved by the third author in [5, Theorem 2.8], but the class of digraphs constructed here is quite different from the ones constructed in [5], which have $r k^{r-s}$ vertices (where $r>s$ ) and automorphism group of order $2 r(k !)^{r}$.

PROOF OF THEOREM 1 . Let $H$ be the group generated by the $s+1$ disjoint permutations

$$
\begin{aligned}
x_{1} & =(1,2, \ldots, k), \\
x_{2} & =(k+1, k+2, \ldots, 2 k), \\
\vdots & \\
x_{s+1} & =(s k+1, s k+2, \ldots,(s+1) k),
\end{aligned}
$$

and then for any positive integer $n$ relatively prime to $s+1$ define

$$
\begin{aligned}
a= & (1, k+1, \ldots, s k+1,(s+1) k+1,(s+1) k+2, \ldots,(s+1) k+n)(2, k+2, \ldots, s k+2) \\
& \cdots(k, 2 k, \ldots,(s+1) k),
\end{aligned}
$$

and let $G$ be the group generated by $H$ and $a$. Clearly $H$ is the direct product of $s+1$ copies of the cyclic group $C_{k}$, acting on $\{1,2, \ldots,(s+1) k\}$ with $s+1$ orbits each of size $k$, and the choice of the permutation $a$ makes $G$ a transitive subgroup of $S_{(s+1) k+n}$. In fact $G$ is doubly transitive and therefore primitive on $\{1,2, \ldots,(s+1) k+n\}$, since the stabilizer in $G$ of the point $(s+1) k+n$ contains each $x_{j}$ (for $\left.1 \leq j \leq s+1\right)$ and $a^{s+1+n}$ as well as the conjugates $a^{-j} x_{1} a^{j}$ of $x_{1}$ (for $1 \leq j \leq s+n$ ), and then since $x_{1}^{-1} a^{-1} x_{s+1} a$ is a 3-cycle, it follows that the group $G$ is either $A_{(s+1) k+n}$ or $S_{(s+1) k+n}$, depending on the parities of $k$ and $s$ and $n$.

(Actually $G=S_{(s+1) k+n}$ unless $k$ is odd and $s+n$ is even.)

Next $a^{-1} \notin H a H$ because every element of $\mathrm{HaH}$ takes the point 1 to one of the points in the set $\{k+1, k+2, \ldots, 2 k\}$ while $a^{-1}$ does not, and so an arc-transitive digraph $\Delta$ may be constructed with the right cosets of $H$ in $G$ as vertices. Also $a^{-1} x_{i} a=x_{i+1}$ for $1 \leq i \leq s$ whereas none of the conjugates of the non-trivial powers 
of $x_{s+1}$ by the permutation $a$ lie in $H$ (because they all move the point $\left.(s+1) k+1\right)$, therefore $H \cap a^{-1} H a$ is the subgroup generated by all the $x_{j}$ other than $x_{1}$, implying that this digraph $\Delta=\Delta(G, H, a)$ is $k$-regular. The intersection of all the conjugates of $H$ in $G$ is trivial, so $G$ acts faithfully on $\Delta$.

Furthermore, for $2 \leq j \leq s$ the stabilizer in $G$ of the $(j-1)-\operatorname{arc}\left(H, H a, H a^{2}, \ldots, H a^{j-1}\right)$ is the intersection of $H$ with its conjugates $a^{-i} H a^{i}$ for $1 \leq i \leq j-1$, which is the subgroup generated by $x_{j}, x_{j+1}, \ldots, x_{s+1}$. The latter group acts transitively on the $k$ out-neighbours of the vertex $H a^{j-1}$ (which are all of the form $H a^{j} h$ with $h \in\left\langle x_{j}\right\rangle$ ), so that $G$ acts transitively on the $j$-arcs of $\Delta$, for $2 \leq j \leq s$. In particular, $\Delta$ is $s$-arctransitive. Also the subgroup $H$ has trivial intersection with its conjugates $a^{-i} H a^{i}$ for $1 \leq i \leq s$, hence the group $G$ acts faithfully on the vertices of $\Delta$, and regularly on the $s$-arcs of $\Delta$.

This completes the proof (since there are infinitely many possible values of $n$ ).

Note that the above proof works for other values of $n$ (having a factor in common with $s+1$, but in those cases the group $G$ is an imprimitive subgroup of $S_{(s+1) k+n}$. Also we have not proved that $G$ is the full automorphism group of $\Delta$, but we suspect this is the case in general.

A further generalization gives rise to the following result.

THEOREM 2. Let $P$ be any finite permutation group which is transitive on a set of size $k$. Then for every positive integer $s$, there are infinitely many examples of a finite $k$-regular digraph $\Delta$ having a group $G$ of automorphisms which acts transitively on the s-arcs (but not on the $(s+1)$-arcs) of $\Delta$, such that also the stabilizer in $G$ of any vertex of $\Delta$ induces the action of $P$ on the set of its $k$ out-neighbours.

PROOF. Without loss of generality suppose $P$ acts on the set $\{1,2, \ldots, k\}$. Let $H$ be the permutation group generated by $s+1$ copies $P_{1}, P_{2}, \ldots, P_{s+1}$ of $P$, such that each copy $P_{i}$ has a single orbit of length $k$, namely $\{(i-1) k+1,(i-1) k+2, \ldots, i k\}$, on which it acts in exactly the same way as $P$ does on $\{1,2, \ldots, k\}$, for $1 \leq i \leq s+1$. Now for any positive integer $n$ relatively prime to $s+1$ define

$$
\begin{aligned}
a= & (1, k+1, \ldots, s k+1,(s+1) k+1,(s+1) k+2, \ldots,(s+1) k+n)(2, k+2, \ldots, s k+2) \\
& \cdots(k, 2 k, \ldots,(s+1) k),
\end{aligned}
$$

and let $G$ be the group generated by $H$ and $a$. In the same way as previously, the choice of the permutation a makes $G$ a 2-transitive subgroup of $S_{(s+1) k+n}$. Also if $x$ is an element of $P$ which moves the point 1 to some other point $p$, say, and if $x_{1}$ and $x_{s+1}$ are the elements of $P_{1}$ and $P_{s+1}$ (respectively) corresponding to $x$, then $x_{1}^{-1} a^{-1} x_{s+1} a=(1,(s+1) k+1, p)$. Thus $G$ is either $A_{(s+1) k+n}$ or $S_{(s+1) k+n}$, depending 
on the nature of $P$ and on the parities of $k$ and $s$ and $n$. Again $a^{-1} \notin H a H$, so that $\Delta=\Delta(G, H, a)$ is an arc-transitive digraph.

Next, while $a^{-1} P_{i} a=P_{i+1}$ for $1 \leq i \leq s$, only those conjugates by $a$ of elements of $P_{s+1}$ fixing the point $s k+1$ lie in $P_{1}$, so $H \cap a^{-1} H a$ is the subgroup stab $P_{P_{1}}(1) \times$ $P_{2} \times \cdots \times P_{s+1}$, of index $k$ in $H$. Thus $\Delta$ is $k$-regular. Also the intersection of all the conjugates of $H$ in $G$ is trivial (as any element in the intersection must fix all the points), so $G$ acts faithfully on $\Delta$. For $2 \leq j \leq s$ the stabilizer in $G$ of the $(j-1)$-arc $\left(H, H a, H a^{2}, \ldots, H a^{j-1}\right)$ is the intersection of $H$ with its conjugates $a^{-i} H^{i}$ for $1 \leq i \leq j-1$, and because this contains the subgroup $P_{j}$, it acts transitively on the $k$ out-neighbours of the vertex $\mathrm{Ha}^{j-1}$ (which are all of the form $\mathrm{Ha}^{j} h$ with the elements $h$ chosen from a transversal for $\operatorname{stab}_{P_{j}}((j-1) k+1)$ in $\left.P_{j}\right)$. Hence the group $G$ acts transitively on the $j$-arcs of $\Delta$, for $2 \leq j \leq s$, and in particular, $\Delta$ is $s$-arc-transitive. Moreover, the action of the stabilizer in $G$ of the $(j-1)$-arc $\left(H, H a, H a^{2}, \ldots, H a^{j-1}\right)$ on the set of $k$ out-neighbours of the vertex $\mathrm{Ha}^{j-1}$ is the same as the action of $P$ on $\{1,2, \ldots, k\}$, for $2 \leq j \leq s$. On the other hand, the stabilizer in $G$ of the $s$-arc $\left(H, H a, H a^{2}, \ldots, H a^{s}\right)$ fixes every vertex of the form $H a^{j}$, because this stabilizer is the direct product of the subgroups $\operatorname{stab}_{P_{j}}((j-1) k+1), 1 \leq j \leq s+1$, and hence $G$ does not act transitively on the $(s+1)$-arcs of $\Delta$.

As there are infinitely many possible values of $n$, this completes the proof.

Generalizing even further, we can use a similar technique to construct an infinite $k$-regular digraph which is $s$-arc-transitive for every positive integer $s$, or 'highly arctransitive' as denoted in [2]. Again several examples of digraphs with these properties were given in [2], but our construction gives a new infinite family of examples, as follows.

Let $H$ be the direct product of a doubly-infinite sequence $\left(\ldots, P_{-2}, P_{-1}, P_{1}, P_{2}, P_{3}, \ldots\right)$ of copies of any transitive permutation group $P$ of degree $k$, with each $P_{-i}$ acting on the set $\{-(i-1) k-1,-(i-1) k-2, \ldots,-i k\}$ and each $P_{i}$ acting on the set $\{(i-1) k+1,(i-1) k+2, \ldots, i k\}$ in the same way as $P$ does on the set $\{1,2, \ldots, k\}$, for $i=1,2,3, \ldots$. Now introduce the extra point 0 and define $a$ as the following permutation of all the integers:

$$
\begin{aligned}
a= & (\ldots,-2 k-1,-k-1,-1,0,1, k+1,2 k+1, \ldots) \\
& (\ldots,-2 k-2,-k-2,-2,2, k+2,2 k+2, \ldots) \\
& \ldots(. .,-3 k,-2 k,-k, k, 2 k, 3 k, \ldots),
\end{aligned}
$$

and let $G$ be the subgroup of the group $\operatorname{Sym}(\mathbb{Z})$ generated by $H$ and $a$.

Just as above, $a^{-1} \notin H a H$, so by the usual argument the right cosets of $H$ in $G$ may be taken as the vertices of a digraph $\Delta$, with the group $G$ acting transitively on the arcs of $\triangle$. Again $H \cap a^{-1} \mathrm{Ha}$ has index $k$ in $H$, for this is the direct product of 
all the $P_{-i}$ (for $i \geq 1$ ) with $\operatorname{stab}_{P_{1}}(1)$ and all the remaining $P_{i}$ (for $i \geq 2$ ), so $\Delta$ is $k$-regular. Also as earlier, but now for every positive integer $s$, the stabilizer in $G$ of the $(s-1)$-arc $\left(H, H a, H a^{2}, \ldots, H a^{s-1}\right)$ acts transitively on the set of $k$ out-neighbours of the vertex $H a^{s-1}$, as this stabilizer is the direct product of all the $P_{-i}$ (for $i \geq 1$ ) and the subgroups $\operatorname{stab}_{P_{i}}((i-1) k+1)$ for $1 \leq i<s$ together with all the remaining $P_{i}$ (for $i \geq s$ ). Thus $\Delta$ is highly arc-transitive, as claimed.

The group $G$ is primitive on $\mathbb{Z}$, and contains all the 3-cycles in $\operatorname{Sym}(\mathbb{Z})$. To see this, first note that if $x_{-1}$ and $x_{1}$ are the elements of $P_{-1}$ and $P_{1}$ (respectively) corresponding to any element $x$ of $P$ which moves the point 1 to some other point $p$, say, then $x_{1}^{-1} a^{-1} x_{-1} a$ is the 3-cycle $(0, p, 1)$. Conjugation of this by $a^{-2} x_{-1} a^{2}$ and then by $a^{-1}$ gives $(0, p, k+p)$ and then $(-1,-p, p)$, and subsequent conjugation of the latter, by $a^{2} x_{1} a^{-2}$ and then by $a$, gives $(-k-p,-p, p)$ and then $(-p, p, k+p)$. Now the stabilizer in $\mathrm{G}$ of the two points 0 and 1 contains not only all the $P_{-i}$ (for $i \geq 1$ ) and $P_{i}$ (for $i \geq 2$ ), but also all the conjugates $a^{-j} P_{-1} a^{j}$ for $j \geq 3$ and all the conjugates $a^{j} P_{1} a^{-j}$ for $j \geq 2$, as well as all the 3-cycles of the form $(-1,-p, p)$ or $(-p, p, k+p)$ for $2 \leq p \leq k$. Finally because the subgroup generated by all of these is transitive on $\mathbb{Z} \backslash\{0,1\}$, it follows that $G$ is 3 -transitive on $\mathbb{Z}$, and also that $G$ contains all possible 3-cycles.

In particular, as every even (finite) permutation can be expressed as a product of 3-cycles, this means $G$ contains every even finitary permutation of the integers. To be more precise, the normal closure $K$ of $H$ in $G$ is either the group of all finitary permutations of $\mathbb{Z}$ or, in the case where $P$ contains no odd permutation on $\{1,2, \ldots, k\}$, the alternating group $\operatorname{Alt}(\mathbb{Z})$. Finally, $G$ is the semi-direct product of this group $K$ by the infinite cyclic group generated by the permutation $a$.

In all of the above variants of Example 1 the subgroup $H$ was a direct product of isomorphic subgroups, and the element $a$ was chosen so as to conjugate each such factor to the next, as far as possible. The next example shows what can happen in other situations where $H$ has two isomorphic but non-conjugate subgroups (analogous to $\langle x\rangle$ and $\langle y\rangle$ in Example 1).

Example 2. The alternating group $A_{6}$ (of order 360 ) has two classes of maximal subgroups of index 6 , each isomorphic to the simple group of order 60 . One way to see this is to consider the three permutations $(1,2,3,4,5),(2,3)(4,5)$ and $(1,6)(2,5)$. The first two generate a natural $A_{5}$ (fixing the point 6 ), while the first and the third correspond to the permutations induced by the linear fractional transformations $z \mapsto$ $z+1$ and $z \mapsto(z-2) /(z-1)$ of the projective line over the field $\mathbb{Z}_{5}$ (with the point 5 relabelled as 0 and the point 6 relabelled as $\infty$ ), giving a transitive action of the group $P S L_{2}(5)$ on six points. Note that in each case we have a pair $(u, v)$ of generators satisfying the relations $u^{5}=v^{2}=(u v)^{3}=1$; in fact these are defining relations for the group $A_{5}$ (see [4]). 
Now although the second subgroup is not conjugate to the first one within $A_{6}$ or even $S_{6}$ (their orbits having different sizes), it is not difficult to embed $A_{6}$ into the symmetric group $S_{12}$ in such a way that the images of these two subgroups become conjugates of each other within this larger group, for instance as follows.

Define $u=(1,2,3,4,5)(7,8,9,10,11), x=(2,3)(4,5)(7,12)(8,11)$, and $y=$ $(1,6)(2,5)(8,9)(10,11)$, and let $K=\langle u, x\rangle, L=\langle u, y\rangle$, and $H=\langle u, x, y\rangle$. The latter group has two orbits $\{1,2,3,4,5,6\}$ and $\{7,8,9,10,11,12\}$, such that the actions of $K$ on the first orbit and of $L$ on the second both duplicate the natural action of $A_{5}$ (fixing one point) described above, while the actions of $K$ on the second and of $L$ on the first duplicate the transitive action of $A_{5}$ on six points. It follows that $K \cong L \cong A_{5}$ and $H \cong A_{6}$. Furthermore, if $c$ is the permutation $(1,7)(2,8)(3,9)(4,10)(5,11)(6,12)$, then $c^{-1} u c=u$ and $c^{-1} x c=y$, so that $c^{-1} K c=L$; that is, $K$ and $L$ are conjugate in $S_{12}$.

Suppose now that we add an odd number of extra points, say $13,14,15, \ldots, n$ (where $n$ is odd), and let $a=(1,7)(2,8)(3,9)(4,10)(5,11)(6,13,14,15, \ldots, n, 12)$ and define $G=\langle u, x, y, a\rangle$ within the symmetric group $S_{n}$. Then we have the same sort of picture as in Example 1.

Note that $G$ is doubly-transitive and therefore primitive, as the stabilizer in $G$ of the point $n$ contains $u, x, y, a^{n-10}$ along with $a^{-j} x a^{j}$ for $1 \leq j \leq n-13$, and in fact $G=S_{n}$ because $y^{-1} a^{-2} y a^{2}$ is a 3 -cycle (fixing the other $n-3$ points). Also $a^{-1} \notin H a H$, since $a^{-1}$ takes the point 13 to the point 6 , while every element of the double coset $H a H$ takes 13 to one of the points of the set $\{7,8,9,10,11,12\}$ if $n=13$, or to 14 if $n>13$. This time $a^{-1} u a=u$ and $a^{-1} x a=y$, so that $a^{-1} K a=L$, but $a^{-1} y a \notin H$ since $a^{-1} y a$ moves the point 13. It follows that $H \cap a^{-1} H a=\langle u, y\rangle=L$, which has index 6 in $H$, and then the intersection of all the conjugates of $H$ in $G$ is trivial, because $H$ is a simple group (isomorphic to $A_{6}$ ).

The digraph $\Delta(G, H, a)$ in this case has $n ! / 360$ vertices and is 6-regular (and connected), with the symmetric group $S_{n}$ as an arc-transitive group of automorphisms, in which the stabilizer of a vertex (namely $H$ ) is isomorphic to $A_{6}$.

In fact $\Delta(G, H, a)$ is 2-arc-transitive, for the stabilizer in $G$ of the $\operatorname{arc}(H, H a)$ is the subgroup $H \cap a^{-1} H a$, which is $L$, and the six in-neighbours of the vertex $H$ are all of the form $H a^{-1} h$ with $h \in L$ (noting that $a^{-1} h \in H a^{-1}$ whenever $h \in K$ ), and these vertices are permuted transitively among themselves by $L$. In particular, this action of the stabilizer of the $\operatorname{arc}(H, H a)$ on the six in-neighbours of $H$ is the same as the usual action of $P S L_{2}(5)$ on the projective line over the field $\mathbb{Z}_{5}$. On the other hand, the other five out-neighbours of $H$ are those vertices of the form $\mathrm{Hah}$ with $h \in K \backslash L$ (since $a h \in H a$ whenever $h \in L$ ), and these are also permuted transitively among themselves by $L$, even by $\langle u\rangle$. To see this, note that in terms of the original permutations the image of the set $\{7,8,9,10,11,12\}$ under any element of a coset $H a h$ is uniquely determined by the effect $h$ has on the point 6 . Thus the stabilizer of 
the arc $(\mathrm{H}, \mathrm{Ha})$ induces the natural action of $A_{5}$ on the other five out-neighbours of $H$.

Again this method works just as well with the addition of an even number of extra points, but the group $G$ is an imprimitive subgroup of $S_{n}$ in those cases. The smallest digraph that results is one on 70560 vertices, coming from the case $n=14$ (with the group $G$ containing the direct product $A_{7} \times A_{7}$ as a subgroup of index 4). A much smaller example will be constructed in the next section.

A similar approach works also for the Mathieu group $M_{12}$ and its two classes of maximal subgroups isomorphic to $M_{11}$. Take

$$
\begin{aligned}
& u=(1,2,3,4,5,6,7,8,9,10,11)(13,23,22,21,20,19,18,17,16,15,14), \\
& x=(1,4)(3,11)(5,9)(7,10)(13,24)(14,20)(19,21)(22,23), \quad \text { and } \\
& y=(1,12)(2,8)(7,9)(10,11)(13,16)(15,23)(17,21)(19,22) .
\end{aligned}
$$

Then the group $H$ generated by $u, x$ and $y$ is isomorphic to $M_{12}$ (with two orbits of size 12), and its subgroups $K=\langle u, x\rangle$ and $L=\langle u, y\rangle$ are non-conjugate subgroups both of which are isomorphic to $M_{11}$ (and having orbits of size 1,11 and 12). These assertions may be easily verified, using the CAYLEY system [3] for instance. Next for any integer $n \geq 25$ define $a=(1,13)(2,14) \cdots(10,22)(11,23)(12,25,26, \ldots, n, 24)$, and let $G=\langle H, a\rangle$.

The same arguments as used in the $A_{5}$ and $A_{6}$ case apply here, except that the element $u$ is inverted (by conjugation) by the element $a$, rather than centralized by $a$, the reason being that every 11-element of $M_{12}$ is self-centralizing in Aut $M_{12}$ (whereas a 5-cycle in $A_{6}$ can be centralized also by an outer automorphism of $S_{6}$ ). Again $H \cap a^{-1} H a=\langle u, y\rangle=L$, which has index 12 in $H$ this time, and so the digraph $\Delta(G, H, a)$ in this case is 12-regular, with either the symmetric group $S_{n}$ or some imprimitive subgroup of $S_{n}$ (depending on whether $n$ is odd or even) as a group of automorphisms acting transitively on its 2-arcs. Also the stabilizer of any 1-arc, say $(v, w)$, acts on the other 11 out-neighbours of $v$ in the same way that $K$ does on the set $\{1,2, \ldots, 11\}$, but on the set of 12 in-neighbours of $v$ in the same way that $K$ does on the set $\{13,14, \ldots, 24\}$.

\section{3. 'Doubling' constructions}

In this section we consider an alternative (but similarly easy) method of construction, in which permutation representations are simply duplicated rather than modified by adding extra points.

Example 3. For any integer $k \geq 2$, let $H$ be the subgroup of $S_{4 k}$ which is generated by the permutations $x=(1,2, \ldots, k)(k+1, k+2, \ldots, 2 k)$ and $y=$ 
$(2 k+1,2 k+2, \ldots, 3 k)(3 k+1,3 k+2, \ldots, 4 k)$. Note that just as in Example $1, H$ is the direct product of two cyclic groups of order $k$, but here each factor has two orbits of size $k$, one of which is like a duplicate of the other. Next define $a=(1,2 k+1, k+$ $1,3 k+1)(2,2 k+2)(3,2 k+3) \cdots(k, 3 k)(k+2,3 k+2)(k+3,3 k+3) \cdots(2 k, 4 k)$, and let $G$ be the group generated by $x, y$ and $a$.

The choice of $a$ ensures that $a^{-1} \notin H a H$, for $a^{-1}$ takes the point 1 to the point $3 k+1$ while every element of the double coset $\mathrm{HaH}$ takes the point 1 to one of the points of the set $\{2 k+1,2 k+2, \ldots, 3 k\}$. Also $a^{-1} x a=y$, while on the other hand $a^{-1} x^{i} y^{j} a \notin H$ whenever $y^{j}$ is non-trivial, again because in that case $a^{-1} x^{i} y^{j} a$ takes the point 1 to one of the points of the set $\{2 k+2,2 k+3, \ldots, 3 k\}$. Thus $H \cap a^{-1} H a=\langle y\rangle$, which has index $k$ in $H$, and the subgroup $H \cap a^{-1} H a \cap a^{-2} H a^{2}$ is trivial.

By the general construction given in Section $1, \Delta(G, H, a)$ is a connected $k$-regular digraph, with $G$ as an arc-regular group of automorphisms.

The choice of $a$ also makes the group $G$ a transitive but imprimitive subgroup of $S_{4 k}$, with the sets $\{1,2, \ldots, 2 k\}$ and $\{2 k+1,2 k+2, \ldots, 4 k\}$ as blocks of imprimitivity (preserved by $x$ and $y$, and interchanged by $a$ ). The stabilizer of each of these blocks is the index 2 subgroup $K$ generated by $H$ and $a^{2}$ and their conjugates, and this group also acts transitively but imprimitively on each block. On one hand the blocks $\{1, k+1\},\{2, k+2\}, \ldots,\{k, 2 k\}$ are fixed by each of $y$ and $a^{2}$ and cyclically permuted by $x$, while on the other hand the blocks $\{2 k+1,3 k+1\},\{2 k+2,3 k+2\}, \ldots,\{3 k, 4 k\}$ are fixed by each of $x$ and $a^{2}$ and cyclically permuted by $y$. The stabilizer in $G$ of all these blocks contains both the double transpositions $x^{-1} a^{-1} y a=(1, k+1)(2, k+2)$ and $y^{-1} a x a^{-1}=(2 k+1,3 k+1)(2 k+2,3 k+2)$ and the double transposition $a^{2}=(1, k+1)(2 k+1,3 k+1)$, and hence (after conjugation of these by $x$ and $y$ and so on) all the products of an even number of the transpositions $s_{i}=(i, k+i)$ for $1 \leq i \leq k$ and $t_{i}=(i, k+i)$ for $2 k+1 \leq i \leq 3 k$.

In particular, as every permutation in $K$ is even, this means that $K$ is a subgroup of index 2 in the direct product of two copies of an extension of an elementary Abelian group of order $2^{k}$ by the cyclic group $C_{k}$ (one copy acting on each of the two large blocks of size $2 k$ ), and therefore $G$ is a group of order $\frac{1}{2}\left(2^{k} k\right)^{2} 2$, that is, $2^{2 k} k^{2}$, and $\Delta(G, H, a)$ has $2^{2 k}$ vertices.

Again this example can be generalized, by taking $H$ as the direct product of more than two cyclic groups of the same order, and/or by using any finite transitive permutation group of degree $k$ instead of the cyclic one, as we show below.

For any integers $k \geq 2$ and $s \geq 1$, and for any transitive subgroup $P$ of the group $S_{k}$, let $H$ be the permutation group generated by $s+1$ copies $P_{1}, P_{2}, \ldots, P_{s+1}$ of $P$, such that each copy $P_{i}$ has two orbits of length $k$, namely $\{2(i-1) k+1,2(i-1) k+$ $2, \ldots,(2 i-1) k\}$ and $\{(2 i-1) k+1,(2 i-1) k+2, \ldots, 2 i k\}$, on each of which it acts 
in exactly the same way as $P$ does on $\{1,2, \ldots, k\}$, for $1 \leq i \leq s+1$. Next define

$$
\begin{aligned}
a= & (1,2 k+1, \ldots, 2 s k+1, k+1,3 k+1, \ldots,(2 s+1) k+1)(2,2 k+2, \ldots, 2 s k+2) \\
& \ldots(k, 3 k, \ldots,(2 s+1) k)(k+2,3 k+2, \ldots,(2 s+1) k+2) \ldots(2 k, 4 k, \ldots, 2(s+1) k),
\end{aligned}
$$

and let $G$ be the group generated by $H$ and $a$.

Just as before, $a^{-1} \notin H a H$, so this gives us an arc-transitive digraph $\Delta=$ $\Delta(G, H, a)$. Also $a^{-1} P_{i} a=P_{i+1}$ for $1 \leq i \leq s$, while only the conjugates by $a$ of those elements of $P_{s+1}$ fixing the point $2 s k+1$ (and then the point $(2 s+1) k+1$ as well) are in $P_{1}$, so $H \cap a^{-1} H a$ is the subgroup $\operatorname{stab}_{P_{1}}(1) \times P_{2} \times \ldots \times P_{s+1}$, of index $k$ in $H$. Thus $\Delta$ is $k$-regular. Moreover, the intersection of all the conjugates of $H$ in $G$ is trivial (for the same reasons as earlier), so $G$ acts faithfully on $\Delta$. Finally, by the same argument as the one used in the proof of Theorem 2, the stabilizer in $G$ of the $(j-1)-\operatorname{arc}\left(H, H a, H a^{2}, \ldots, H a^{j-1}\right)$ acts transitively on the $k$ out-neighbours of the vertex $\mathrm{Ha}^{j-1}$ (which are all of the form $\mathrm{Ha}^{j} h$ with the elements $h$ chosen from a transversal for $\operatorname{stab}_{P_{j}}(2(j-1) k+1)$ in $\left.P_{j}\right)$, for $2 \leq j \leq s$. Thus $\Delta$ is $s$-arc-transitive, and in fact the stabilizer in $G$ of any vertex of $\Delta$ induces the action of $P$ on the set of its $k$ out-neighbours.

The group $G$ is a transitive but imprimitive subgroup of the symmetric group $S_{2(s+1) k}$, with $s+1$ blocks of size $2 k$ that are preserved by every element of $H$ and cyclically permuted by $a$. The stabilizer of each of these blocks is the subgroup $K$ generated by $H$ and $a^{s+1}$ and their conjugates, and then also $K$ acts transitively but imprimitively on each block. For example, on $\{1,2, \ldots, 2 k\}$ this group $K$ permutes the $k$ sets of the form $\{j, j+k\}$ (for $1 \leq j \leq k$ ) amongst themselves in the same way as $P$ acts on $\{1,2, \ldots, k\}$. Furthermore, if $x_{1}$ and $x_{s+1}$ are the elements of $P_{1}$ and $P_{s+1}$ (respectively) corresponding to any element $x$ of $P$ which moves the point 1 , to the point $j$, say, then $x_{1}^{-1} a^{-1} x_{s+1} a$ is the double transposition $d_{j}=(1, k+1)(j, k+j)$. From this it follows that the conjugates of all elements of $H$ generate the direct product of $s+1$ copies of an extension of an elementary Abelian group of order $2^{k-1}$ by the group $P$ (with each copy acting transitively but imprimitively on one of the $s+1$ blocks of size $2 k$ ). Moreover $K$ itself is a semi-direct product of this subgroup by the cyclic subgroup $\left\langle a^{s+1}\right\rangle$ of order 2 . It now follows that the group $G$ has order $\left(2^{k-1}|P|\right)^{s+1} 2(s+1)$, that is $(s+1) s^{k s-s+k}|P|^{s+1}$, and therefore $\Delta$ has $(s+1) 2^{k s-s+k}$ vertices.

Note that the size of $\Delta$ is independent of the choice of the permutation group $P$ (in contrast to the situation following Example 1). In fact the digraph $\Delta$ itself is independent of $P$, that is to say, the same digraph is obtained whatever particular transitive group of degree $k$ is chosen. One way to see this is to notice that $P$ could be taken as the full symmetric group of degree $k$, in which case the corresponding group $G$ has order $\left(2^{k-1} k !\right)^{s+1} 2(s+1)$ and contains every other group arising from 
this construction as a subgroup, and so on. But it turns out there's a much easier way of recognising this fact, because the digraph $\Delta$ is actually a Cayley digraph.

If $N$ is the group generated by $a$ and the double transpositions $d_{j}=(1, j)(k+1$, $k+j$ ) for $2 \leq j \leq k$, then $N$ is an extension by a cyclic group of order $s+1$ of an elementary Abelian group of order $2^{k s-s+k}$ (generated by the involution $a^{s+1}$ and all the conjugates $a^{-i} d_{j} a^{i}$ for $0 \leq i \leq s$ and $2 \leq j \leq k$ ). In particular, $N$ is clearly a complement for the subgroup $H$ in $G$, so the vertices of $\Delta$ are in one-to-one correspondence with the elements of $N$. Now if $H x$ is any vertex of $\Delta$ (with $x \in N$ ), the out-neighbours of $H x$ in $\Delta$ are precisely those cosets of the form $H a h x$ with $h \in H$, indeed with $h \in P_{1}$ since $a h \in H a$ whenever $h \in P_{i}, i \neq 1$. Also if, say, $h$ moves the point 1 to the point $j$, and $g$ is the corresponding element of $P_{s+1}$, then $h^{-1} a^{-1} g a=(1, k+1)(j, k+j)=d_{j}$, so that $\operatorname{Hahx}=\operatorname{Hgad}_{j}^{-1} x=\operatorname{Had}_{j}^{-1} x=$ $\operatorname{Had}_{j} x$. With $d_{1}$ defined as the identity permutation, it follows that $\Delta$ is a Cayley (di-)graph for the group $N$, with generating-set $X=\left\{a d_{j} \mid 1 \leq j \leq k\right\}$.

A similar technique may be used to construct a highly arc-transitive infinite $k$ regular digraph, as in Section 1.

Let $H$ be the direct sum of a doubly-infinite sequence $\left(\ldots, P_{-2}, P_{-1}, P_{0}, P_{1}, P_{2}, \ldots\right)$ of copies of any transitive permutation group $P$ of degree $k$, with each $P_{i}$ acting on the two sets $\{2(i-1) k+1,2(i-1) k+2, \ldots,(2 i-1) k\}$ and $\{(2 i-1) k+1,(2 i-$ $1) k+2, \ldots, 2 i k\}$ in the same way as $\mathrm{P}$ does on the set $\{1,2, \ldots, k\}$, for every $i \in \mathbb{Z}$. Next define $a$ as the following permutation of all the integers:

$$
\begin{aligned}
a= & (\ldots,-3 k+1,-k+1,1,2 k+1,4 k+1, \ldots)(\ldots,-4 k+1,-2 k+1, k+1,3 k+1,5 k+1, \ldots) \\
& (\ldots,-4 k+2,-2 k+2,2,2 k+2,4 k+2, \ldots)(\ldots,-3 k+2,-k+2, k+2,3 k+2, \ldots) \\
& \vdots \\
& (\ldots,-3 k,-k, k, 3 k, 5 k, \ldots)(\ldots,-2 k, 0,2 k, 4 k, \ldots),
\end{aligned}
$$

and let $G$ be the subgroup of the group $\operatorname{Sym}(\mathbb{Z})$ generated by $H$ and $a$.

Just as in all previous cases, the right cosets of $H$ in $G$ may be taken as the vertices of a digraph $\Delta$, with the group $G$ acting transitively on the arcs of $\Delta$, and again $H \cap a^{-1} H a$ has index $k$ in $H$, for this is the direct sum of $\operatorname{stab}_{P_{1}}(1)$ with all the remaining $P_{i}$ (for $i \neq 1$ ), so $\Delta$ is $k$-regular. Also as earlier, but now for every positive integer $s$, the stabilizer in $G$ of the $(s-1)$-arc $\left(H, H a, H a^{2}, \ldots, H a^{s-1}\right)$ acts transitively on the set of $k$ out-neighbours of the vertex $H a^{s-1}$, as this is the direct sum of all the subgroups $\operatorname{stab}_{P_{i}}(2(i-1) k+1)$ for $1 \leq i<s$ together with all the remaining $P_{i}$ (for $i \leq 0$ and for $i \geq s$ ). Thus $\Delta$ is highly arc-transitive, as claimed, and again the same digraph is obtained whatever the choice of $P$. Furthermore, the normal closure $K$ of $H$ in $G$ is contained in the group of all finitary permutations of $\mathbb{Z}$, and $G / K$ is infinite cyclic (generated by $K a$ ). 
We now proceed to see what can happen in the $A_{5}$ and $A_{6}$ case using this technique of 'doubling' a permutation representation.

\section{Example 4. Let}

$$
\begin{aligned}
& u=(1,2,3,4,5)(7,8,9,10,11)(13,14,15,16,17)(19,20,21,22,23), \\
& x=(2,3)(4,5)(8,9)(10,11)(13,18)(14,17)(19,24)(20,23), \\
& y=(1,6)(2,5)(7,12)(8,11)(14,15)(16,17)(20,21)(22,23),
\end{aligned}
$$

and define $K=\langle u, x\rangle, L=\langle u, y\rangle$, and $H=\langle u, x, y\rangle$. The latter group has four orbits $\{1, \ldots, 6\},\{7, \ldots, 12\},\{13, \ldots, 18\}$ and $\{19, \ldots, 24\}$, such that the actions of $K$ on the first two and of $L$ on the last two orbits both duplicate the natural action of $A_{5}$ (fixing one point in each case), while the actions of $K$ on the last two and of $L$ on the first two orbits duplicate the transitive action of $A_{5}$ on six points. In particular, as in Example 2, $K \cong L \cong A_{5}$ and $H \cong A_{6}$. Next let

$$
\begin{aligned}
a= & (1,13)(2,14)(3,15)(4,16)(5,17)(6,24,12,18)(7,19)(8,20)(9,21)(10,22) \\
& (11,23),
\end{aligned}
$$

and define $G=\langle u, x, y, a\rangle$ within the symmetric group $S_{24}$. Then we have the same sort of picture as in Example 3.

First note $a^{-1} \notin H a H$, since $a^{-1}$ takes the point 24 to the point 6 , while every element of the double coset $\mathrm{HaH}$ takes 24 to one of the points of the set $\{7,8,9,10,11,12\}$. Also the choice of $a$ makes $a^{-1} u a=u$ and $a^{-1} x a=y$, so that $a^{-1} K a=L$, but $a^{-1} y a \notin H$ since $a^{-1} y a$ moves the point 13 to the point 24 . It follows that $H \cap a^{-1} H a=\langle u, y\rangle=L$, which has index 6 in $H$, and again the intersection of all the conjugates of $H$ in $G$ is trivial.

The group $G$ in this case is a transitive but imprimitive subgroup of $S_{24}$, with two blocks $\{1,2, \ldots, 12\}$ and $\{13,14, \ldots, 24\}$ that are preserved by each of $u, x$ and $y$, and interchanged by the permutation $a$. Stabilizing these two blocks is the subgroup generated by $H$ and $a^{2}$ and their conjugates. This may be seen to be an extension by $A_{6}$ of an elementary Abelian group of order $2^{11}$, containing all the products of an even number of the transpositions $\{1,7\},\{2,8\}, \ldots,\{6,12\}$ and $\{13,19\},\{14,20\}, \ldots,\{18,24\}$. For example, it contains the double transposition $x^{-1} a^{-1} y a=(13,19)(18,24)$. It follows that $G$ is a group of order $2^{11} \times 360 \times 2$, with the subgroup $H$ having index $2^{12}$, so the digraph $\Delta(G, H, a)$ has only 4096 vertices.

Of course $\Delta(G, H, a)$ is 6-regular, the stabilizer in $G$ of any vertex is isomorphic to $A_{6}$, and, just as in Example 2, the stabilizer in $G$ of the $\operatorname{arc}(H, H a)$ is the subgroup $H \cap a^{-1} H a$, namely $L$, and this acts on the other five out-neighbours of the vertex $H$ in the same way as a natural $A_{5}$, while acting on the six in-neighbours of $H$ in the same way as $P S L_{2}(5)$ acts on the projective line over the field $\mathbb{Z}_{5}$. 
The same approach works also in the case of $M_{11}$ and $M_{12}$, giving a digraph on $2^{24}$ vertices with the same properties as those described at the end of Section 2.

Indeed both this type of 'doubling' construction and the 'extra point' technique introduced earlier can obviously be applied in any situation where a given finite group contains two or more isomorphic but non-conjugate subgroups. In Examples 1 and 3 (and their generalizations) the group $H$ is a direct product and the relevant subgroups are its factors, while in Examples 2 and 4 (and their analogues) these subgroups are non-conjugate maximal subgroups of a simple group.

Also this technique of duplicating permutation representations can obviously be taken further, for example by 'tripling' instead of doubling, and a whole raft of possibilities arise.

Unfortunately we note that the question raised in [2] about the existence of a vertexprimitive 2-arc-transitive digraph remains unanswered. Even though our constructions are quite general we have so far found only examples which are vertex-imprimitive.

Note also that in all of our constructions of infinite highly arc-transitive digraphs the group $G$ acting on the digraph has a normal subgroup $K$ containing the vertexstabilizers, such that $G / K$ is an infinite cyclic group. In [2, Question 2] it was asked whether this must always be the case when the in- and out-degrees are finite. It was proved in [6] that this must be the case when the (finite) in- and out-degrees are unequal, but the question is unanswered for regular infinite highly arc-transitive digraphs of finite valency.

\section{Miscellany}

We conclude this paper with a couple of other (miscellaneous) constructions which we have also discovered during the course of our investigations.

Example 5. The group $P S L_{2}(11)$ in its natural action on the projective line over the field $\mathbb{Z}_{11}$ may be generated by the four linear fractional transformations $z \mapsto-1 / z$, $z \mapsto(z+5) /(4 z-1), z \mapsto(z+3) /(3 z-1)$, and $z \mapsto(z+1) /(z-1)$, which induce the respective permutations

$$
\begin{aligned}
p & =(0, \infty)(1,10)(2,5)(3,7)(4,8)(6,9), \\
q & =(0,6)(1,2)(3, \infty)(4,5)(7,9)(8,10), \\
r & =(0,8)(1,2)(3,9)(4, \infty)(5,10)(6,7), \text { and } \\
s & =(0,10)(1, \infty)(2,3)(4,9)(5,7)(6,8) .
\end{aligned}
$$

Let $H$ be the group generated by these four permutations, so that $H$ is isomorphic to $P S L_{2}(11)$ in its natural transitive action on 12 points. Now, because $p, q$ and $r$ satisfy the relations $p^{2}=q^{2}=r^{2}=(p q)^{3}=(q r)^{5}=(p r)^{2}=(p q r)^{5}=1$, the 
subgroup $K=\langle p, q, r\rangle$ is isomorphic to $A_{5}$ (cf. the group $G^{3,5.5}$ in [4]). Similarly, because $q^{2}=r^{2}=s^{2}=(q r)^{5}=(r s)^{3}=(q s)^{2}=(q r s)^{5}=1$, also the subgroup $L=\langle q, r, s\rangle$ is isomorphic to $A_{5}$.

Next define a new permutation $a=(3,6,10, \infty, 7,8,4,9,11,5)$, and let $G$ be the group generated by $H$ and $a$. This choice of $a$ is made so that $a^{-1} p a=q$, $a^{-1} q a=r$, and $a^{-1} r a=s$. On the other hand, $a^{-1} s a \notin H$, since for example the product of $a^{-1} s a$ with pqrs has order 14 and hence is not in $H$. In particular, $H \cap a^{-1} H a=\langle q, r, s\rangle=L$, which is a maximal subgroup (of index 11) in $H$.

The group $G$ itself is primitive on $\{0,1,2, \ldots, 10, \infty\}$, and contains not only the odd permutation $a$, but also the 7-cycle $\left(a^{-1} \text { sa pqrs }\right)^{2}=(0,1,4,3,6,9, \infty)$, so that by Jordan's theorem [8, Theorem 13.9], we find $G$ is isomorphic to $S_{12}$.

In the canonical action of $G$ on right cosets of $H$, the subgroup $L$ fixes the coset $H a$, and has a single orbit of length 10 on the remaining cosets of the form $H a h$ with $h \in H$, since if $H a p x=H a p y$ with $x, y \in L$ then $a p x y^{-1} p a^{-1} \in H$ and therefore $p x y^{-1} p \in a^{-1} H a \cap p L p$, which is easily found to be the subgroup $\langle r, s\rangle$, of index 10 in $L$. On the other hand, $L$ has two orbits on cosets of the form $\mathrm{Ha}^{-1} h$ with $h \in H$, one of length 6 consisting of all those of the form $\mathrm{Ha}^{-1} x$ with $x \in L$, and another of length 5 made up of those remaining, all of the form $\mathrm{Ha}^{-1} t x$ with $t=s r q p$ (for instance) and $x \in L$. (Details are left to the reader.)

As a consequence, the actions of $L$ on the right cosets of $\mathrm{H}$ in $\mathrm{HaH}$ and in $\mathrm{Ha}^{-1} \mathrm{H}$ are distinct, implying that $\mathrm{HaH} \neq \mathrm{Ha}^{-1} \mathrm{H}$. Thus we have an 11-regular arc-transitive digraph $\Delta$ on 725760 vertices (by our usual construction), with the group $G \cong S_{12}$ acting on $\Delta$ in such a way that the stabilizer in $G$ of an $\operatorname{arc}(v, w)$ in $\Delta$ is isomorphic to $P S L_{2}(11)$, and this group has one orbit of length 10 on the other out-neighbours of $v$, and orbits of lengths 5 and 6 on the 11 in-neighbours of $v$. In particular, $G$ has two orbits on 2-arcs.

Example 6. The intersection of two non-conjugate subgroups of index 6 in the group $A_{6}$ is a dihedral group of order 10, of index 36. Using CAYLEY (for instance) it is not difficult to calculate the permutations induced by generators given for $A_{6}$ at the beginning of Example 2 on the right cosets of this intersection. In fact these may be taken as

$$
\begin{aligned}
u= & (2,3,4,5,6)(7,8,9,10,11)(12,13,14,15,16)(17,18,19,20,21) \\
& (22,23,24,25,26)(27,28,29,30,31)(32,33,34,35,36), \\
x= & (1,2)(4,5)(7,12)(8,17)(9,27)(10,32)(11,22)(13,23)(14,35)(15,29) \\
& (16,21)(18,33)(19,25)(20,34)(24,30)(26,31), \quad \text { and } \\
y= & (1,7)(2,12)(3,25)(4,36)(5,28)(6,19)(9,10)(13,20)(14,31)(15,33) \\
& (16,24)(17,22)(18,35)(21,34)(23,30)(26,29),
\end{aligned}
$$


with $u$ and $(x y)^{2}$ generating the (dihedral) stabilizer of the point 1 .

Let $H=\langle u, x, y\rangle$, and $K=\langle u, x\rangle$ and $L=\langle u, y\rangle$, so that $H \cong A_{6}$ while $K \cong L \cong A_{5}$. Note that each of $K$ and $L$ has two orbits on $\{1,2, \ldots, 36\}:$ one of length 6 containing the point 1 , and another of length 30 . Those of length 6 are $\{1,2,3,4,5,6\}$ and $\{1,7,8,9,10,11\}$ respectively, and the actions of $K$ and $L$ on the other orbits are easily seen in the diagrams below.
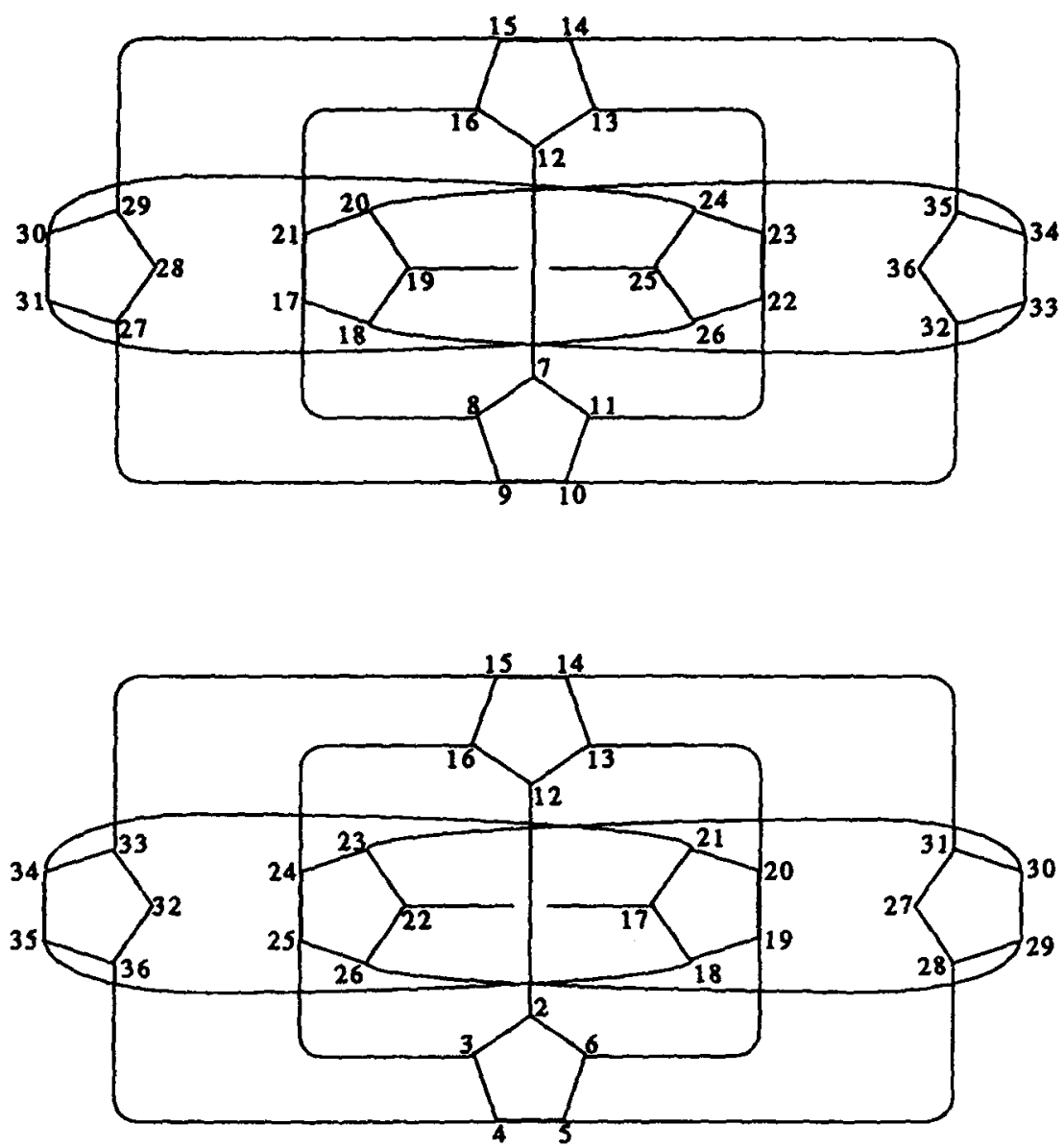

Here the cycles of the permutation $u$ are depicted by pentagons (whose vertices are shifted anti-clockwise by $u$ ), and the points at the ends of all remaining edges are interchanged by $x$ and by $y$ in the respective cases.

Now in this representation of $A_{6}$, there are two possibilities for a permutation $a$ of the same 36 points which centralizes $u$ and conjugates $x$ to $y$ (as in Example 2). In 
one case $a$ is an involution, fixing the point 1 and interchanging 2 with 7 , and 3 with 8 , and so on, in a way that can be seen from the diagrams by placing one immediately on top of the other. In this case, however, $a^{-1}=a \in H a H$, which is not appropriate for the construction of a digraph. On the other hand, if the first diagram is rotated 180 degrees and then placed on top of the second, we find a second possibility for $a$ is the following:

$$
\begin{aligned}
a= & (2,7,12)(3,8,13)(4,9,14)(5,10,15)(6,11,16)(17,20,18,21,19) \\
& (22,24,26,23,25)(27,31,30,29,28)(32,33,34,35,36) .
\end{aligned}
$$

Again in this case $H \cap a^{-1} H a=L$, because $a^{-1} u a=u$ and $a^{-1} x a=y$, but $a^{-1} y a \notin H$ for the element $\left(a^{-1} y a\right) x u^{2} y$ has order 12 and therefore cannot be in $H$. Moreover, just as in Example 5, the actions of $L$ on right cosets of $H$ in $\mathrm{HaH}$ and in $\mathrm{Ha}^{-1} \mathrm{H}$ are distinct. On the one hand $L$ has two orbits on those of the first type (one being $\{\mathrm{Ha}\}$ by itself and the second consisting of the other five cosets, all of the form $H a x h$ with $h \in L$ ), while on the other hand $L$ acts transitively on the six cosets of the form $H a^{-1} h$ with $h \in H$ (since if $H a^{-1} p=H a^{-1} q$ with $p, q \in L$ then $a^{-1} p q^{-1} a \in H$ and therefore $p q^{-1} \in a H a^{-1} \cap L$, which is easily found to be the subgroup $\left\langle u,(x y)^{2}\right\rangle$, of index 6 in $\left.L\right)$. In particular, $a^{-1} \notin H a H$. Finally we note that the permutation $a^{6} x u$ is made up of a 19-cycle, a 7-cycle, a 5-cycle, a 3-cycle and two fixed points, and then since $\left(a^{6} x u\right)^{105}$ is a single 19-cycle, $G$ must be primitive, and by Jordan's theorem, in fact $G=A_{36}$.

Thus we have yet another example of a 6-regular 2-arc-transitive digraph $\Delta$ with a group of automorphisms having the properties stated in the introduction.

\section{Acknowledgments}

The first and second authors are grateful to the N. Z. Lotteries Board and the N. Z. University Grants Committee for research support, and to the Department of Mathematics \& Statistics at the University of Auckland for sponsoring the visit of the third author to Auckland in 1988.

\section{References}

[1] P. J. Cameron, Structure of sub-orbits in some primitive permutation groups (D. Phil. thesis, University of Oxford, 1971).

[2] P. J. Cameron, C. E. Praeger and N. C. Wormald, 'Infinite highly arc transitive digraphs and universal covering digraphs', Combinatorica 13 (1993), 377-394.

[3] J. J. Cannon, 'An introduction to the group theory language CAYLEY', in: Computational group theory (ed. M. Atkinson) (Academic Press, San Diego CA/London, 1984) pp. 145-183. 
[4] H. S. M. Coxeter and W. O. J. Moser, Generators and relations for discrete groups, 4th edition (Springer, Berlin, 1980).

[5] C. E. Praeger, 'Highly arc transitive digraphs', European J. Combin. 10 (1989), 281-292.

[6] C. E. Praeger, 'On homomorphic images of edge transitive directed graphs', Austral. J. Combin. 3 (1991), 207-210.

[7] R. Weiss, 'The non-existence of 8-transitive graphs', Combinatorica 1 (1981), 309-311.

[8] H. Wielandt, Finite permutation groups (Academic Press, New York, 1964).

Department of Mathematics

University of Auckland

Private Bag 92019, Auckland

New Zealand

Department of Mathematics

University of Western Australia

Nedlands WA 6009

Australia
Department of Mathematics

University of Auckland

Private Bag 92019, Auckland

New Zealand 